\title{
On the Space of Bounded Mean Oscillations
}

\author{
Santosh Ghimire a , Arjan Kumar Sunar b \\ a Dept. of Applied Sciences \& Chemical Engineering, Pulchowk Campus, Institute of Engineering, Tribhuvan University, Nepal \\ ${ }^{\mathrm{b}}$ Kathford International College of Engineering and Management, Tribhuvan University, Nepal \\ Corresponding Authors: ${ }^{a}$ santoshghimire@ioe.edu.np, ${ }^{b}$ aarjan.sunar@kathford.edu.np
}

Received: 2021-01-16

Revised: 2021-01-26

Accepted: 2021-02-19

\begin{abstract}
:
The space of bounded mean oscillations, abbreviated BMO, was first introduced by F. John and L. Nirenberg in 1961 in the context of partial differential equations. Later, C. Fefferman proved that the BMO is the dual space of well-known Hardy space, popularly known as $H^{1}$ space and became the center of attraction for mathematicians. With the help of BMO space, many mathematical phenomenon can be characterized clearly. In this article, we discuss the connections of function of bounded mean oscillations with weight functions, sharp maximal functions and Carleson measure.
\end{abstract}

Keywords: BMO, weight function, maximal function, Carleson measure

\section{Introduction}

The Lebesgue spaces, popularly known as $L_{p}$ spaces, play an important role in Fourier analysis. However, many important classes of operators are not well behaved on the $L_{p}$ spaces particularly in $L_{1}$ and $L_{\infty}$ spaces. Therefore, $L_{1}$ is too large to be the domain of such operators. Similarly, the target space of many canonical operators exceeds $L_{\infty}$. Consequently, $L_{\infty}$ is too small to be the range of such operators. These two spaces are considered as dual of each other in certain sense. The motivation to find substitutes for the spaces $L_{\infty}$ and $L_{1}$ led to the space of bounded mean oscillations and the Hardy space $H^{1}$. The space of bounded mean oscillations is abbreviated BMO space. The functions of bounded mean oscillation was originally introduced by F. John and L. Nirenberg in the context of partial differential equations [1]. This space naturally arises as the class of function whose deviation from their means over cubes is bounded. These spaces turned out to be the "right" spaces to study instead of $L_{1}$ and $L_{\infty}$ respectively. In fact many operators which are ill-behaved on $L_{1}$ or $L_{\infty}$ are bounded on $H^{1}$ and on $\mathrm{BMO}$. Thus the space of BMO is strictly including the $L_{\infty}$ space and is a good extension of the Lebesgue spaces $L_{p}$ with $1<p<\infty$ from a point of view of Harmonic Analysis. Thus the space of bounded mean oscillation turns out to be a natural substitute for
$L^{\infty}\left(\mathbb{R}^{n}\right)$ in harmonic analysis. Every bounded function belongs to BMO, but there exist unbounded functions with bounded mean oscillations. Such a functions typically blow up logarithmically as shown by the John-Nirenberg theorem[1]. The relevance of BMO is attested by the fact that the classical singular integral operators fail to map $L^{\infty}\left(\mathbb{R}^{n}\right)$ to $L^{\infty}\left(\mathbb{R}^{n}\right)$, but instead they map $L^{\infty}\left(\mathbb{R}^{n}\right)$ to BMO. Moreover, BMO is the dual space of the Hardy space $H^{1}$. This observation was announced by $\mathrm{C}$. Fefferman in [1] and then proved in [2]. We can show that every bounded function is in BMO. This gives that $L^{\infty} \subset B M O$. But there exist functions which are in BMO but not in $L^{\infty}$. A famous example for this is $f(x)=\log |x|$ which is in $B M O\left(\mathbb{R}^{n}\right)$ but not in $L^{\infty}\left(\mathbb{R}^{n}\right)$. But BMO is not much larger than $L^{\infty}$ space. Also BMO scales the same way as $L^{\infty}$ : if $f(x)$ is in BMO then so is $f(\lambda x)$ for any $\lambda>0$ with the same BMO norm. The space of BMO is much more needed in the study of various situations such as boundedness of Calderon-Zygmund operators, real interpolation, Carleson measure, study of paraproducts etc. to just name a few. BMO also plays a central role in the regularity theory for non linear partial differential equations. In this article, we discuss the relations of functions of BMO with weight function, sharp maximal functions and Carleson measure. In order to do this, we first state some definitions. We begin with the definition of functions of bounded mean oscillations. 
Definition [Functions of bounded mean oscillations]

A real-valued locally integrable function $f(x)$ defined on $\mathbb{R}^{n}$ is said to in BMO, the space of functions of bounded mean oscillation, if for any measurable set $Q \subset \mathbb{R}^{n}$, we have:

$$
\sup _{Q} \frac{1}{|Q|} \int_{Q}\left|f(x)-f_{Q}\right| d x<\infty
$$

where the supremum is taken over all cubes $Q$ in $\mathbb{R}^{n}$ with sides of the cube parallel to coordinate axes, $|Q|$ denotes the measure of $Q$, and where $f_{Q}$ is the average value of $f$ on the cube $Q$ and is defined as:

$$
f_{Q}=\frac{1}{|Q|} \int_{Q} f(x) d x
$$

BMO norm a function $\mathrm{f}$, denoted by $\|f\|_{B M O}$, is defined as:

$$
\|f\|_{B M O}=\sup _{Q} \frac{1}{|Q|} \int_{Q}\left|f(x)-f_{Q}\right| d x
$$

From the definition, we note that if $\mathrm{f}, \mathrm{g}$ are two functions in $B M O\left(\mathbb{R}^{n}\right)$ and $\lambda_{1}, \lambda_{2} \in \mathbb{R}$, then one can easily show that the linear combination $\lambda_{1} f+\lambda_{2} g$ is also in $B M O\left(\mathbb{R}^{n}\right)$. This shows that space BMO is a linear space. Moreover, we also note that

$$
\|\mathrm{f}+\mathrm{g}\|_{B M O} \leq\|\mathrm{f}\|_{B M O}+\|\mathrm{g}\|_{B M O}
$$

and

$$
\left\|\lambda_{1} \mathrm{f}\right\|_{B M O} \leq\left|\lambda_{1}\right|\|\mathrm{f}\|_{B M O}
$$

If $\|\mathrm{f}\|_{B M O}=0$, then $\int_{Q}\left|f(x)-f_{Q}\right| d x=0$. Then $\mathrm{f}$ has to be equal to its average $f_{Q}$ on every cube, say $Q_{N}=[-N, N]^{n}$ We note that the cube $Q_{N}$ is contained in $\left.Q_{(} N+1\right)=[-(N+1), N+1]^{n}$. This forces that $\left.\left.f_{(} Q_{N}\right)=f_{(}\left(Q_{(} N+1\right)\right)$. Thus we have if $\|\mathrm{f}\|_{B M O}=0$, then $\mathrm{f}$ is a.e. equal to a constant. Consequently, we have the $\|\cdot\|_{B} M O$ is not a norm. Note that BMO norm of constant is zero. This gives $f$ and $f+C$ have the same $\mathrm{BMO}$ norm with $\mathrm{C}$ being a constant. In the discussion that follows after this we take $\|\cdot\|_{B} M O$ as a norm even though it is a semi norm when there is no possibility of confusion.

With the introduction of maximal functions one can better understand the concept of averages of functions in analysis. Maximal functions are widely used in differentiation theory in analysis. Roughly the maximal function is defined as the largest value of the averages of functions over all possible balls which contain a fixed point. Maximal functions appear in many forms. The most important of these is the Hardy-Littlewood maximal function. We now define Hardy-Littlewood maximal function:
Let $f \in L^{p}\left(\mathbb{R}^{n}\right), 1 \leq p<\infty$.

and

$$
M f(x)=\sup _{r>0} \frac{1}{|B(x, r)|} \int_{B(x, r)}|f(y)| d y
$$

Then $M f$ is called the Hardy-Littlewood maximal function of $f$. In the definition of maximal function, all the balls are centered at the point $x$ Therefore, this is centered Hardy-Littlewood maximal function. Similarly, we can define uncentered Hardy-Littlewood maximal as:

$$
M^{u} f(x)=\sup _{r>0, x \in B} \frac{1}{|B|} \int_{B}|f(y)| d y
$$

\section{Definition [Weight]}

A locally integrable function on $\mathbb{R}^{n}$ that takes values in the interval $(0, \infty)$ almost everywhere is called a weight. So by definition a weight function can be zero or infinity only on a set whose Lebesgue measure is zero. We use the notation $w(E)=\int_{E} w(x) d x$ to denote the w-measure of the set $\mathrm{E}$ and we reserve the notation $L^{p}\left(\mathbb{R}^{n}, w\right)$ or $L^{p}(w)$ for the weighted $L^{p}$ spaces. We note that $w(E)<$ $\infty$ for all sets $\mathrm{E}$ contained in some ball since the weights are locally integrable functions.

Definition: A function $w(x)>0$ is called an $A_{1}$ weight if there is a constant $C_{1}>0$ such that $M(w)(x) \leq C_{1} w(x)$ where $M(w)$ is uncentered Hardy-Littlewood maximal function. If $w$ is an $A_{1}$ weight, then the quantity (which is finite) given by:

$$
[w]_{A_{1}}=\sup _{\mathrm{Q} \text { cubes in } \mathbb{R}^{n}}\left(\frac{1}{|Q|} \int_{Q}|w(t)| d t\right)\left\|w^{-1}\right\|_{L^{\infty}(Q)}
$$

is called the A1 Muckenhoupt characteristic constant of w or simply $A_{1}$ characteristic constant of w. We first begin with the relation between the functions of $\mathrm{BMO}$ and weight function. For more about the weight functions, please refer [3].

\section{BMO and Weight Function}

The theory of weights play an important role in various fields such as extrapolation theory, vector-valued inequalities and estimates for certain class of non linear differential equation. Moreover, they are very useful in the study of boundary value problem for Laplace's equation in Lipschitz domains. Some of the theory of Muckenhoupt's $A_{p}$ weights and weighted norm inequalities results can be used to give characterizations of BMO function. One of the very important property 
of BMO functions is the John-Nirenberg Inequality given by the theorem of John-Nirenberg stated as follows:

Theorem [John-Nirenberg Theorem] For all functions $\mathrm{f}$ in the space of BMO defined on $\mathbb{R}^{n}$; for all cubes $Q$ and $\alpha>0$, we have

$$
\left|\left\{x:\left|f(x)-A v g_{Q} f\right|>\alpha\right\}\right| \leq e|Q| e^{-A \alpha\|f\|_{\text {BMO }}}
$$

with $A=\left(2^{n} e\right)^{-1}$.

For the proof the theorem, please refer, page 124 of [3]. An immediate consequence of the John-Nirenberg inequality is the $\mathrm{p}$-invariance property for any $1<p<$ $\infty$,

$$
\|f\|_{B M O} \approx \sup _{Q}\left(\frac{1}{|Q|} \int_{Q}\left|f(x)-f_{Q}\right|^{p} d x\right)^{\frac{1}{p}}
$$

Moreover, using the John-Nirenberg inequality, for any $f \in B M O$, the function $e^{|f(x)| / \rho}$ is locally integrable for some appropriate constant $\rho>0$. Now we note the following result which can be easily proved: Let $V(t)$ be a real valued locally integrable function on $\mathbb{R}^{n}$ and let $1<p<\infty$. Then the function $e^{V(t)} \in A_{p}$ if and only if two conditions are satisfied for some constant $c<\infty$.

$$
\begin{array}{ll}
\text { a. } & \sup _{Q} \frac{1}{|Q|} \int_{Q} e^{V(t)-V_{Q}} d t \leq C \\
\text { b. } & \sup _{Q} \frac{1}{|Q|} \int_{Q} e^{-\left[V(t)-V_{Q}\right]^{\frac{1}{p-1}}} d t \leq C
\end{array}
$$

Using the above relation, one can establish a deep connection between the weight functions and BMO functions. Precisely, logarithm of any $A_{2}$ function is a $\mathrm{BMO}$ function and every BMO function is equal to a constant multiple of the logarithm of an $A_{2}$ weight function. Moreover, we show that logarithm of any $A_{p}$ weight function for $1<p<\infty$ is a BMO function. For more details, please refer [4]. In addition to this connection, there is another relation between weight functions and functions in BMO. For this, we recall the weighted BMO space. Similar to weighted Lebesgue spaces, one can define weighted BMO space, denoted by $B M O_{w}$, as the collections of $w$ locally integrable functions $f$ such that

$$
\|f\|_{B M O_{w}}=\sup _{Q \subset \mathbb{R}^{n}} \frac{1}{w(Q)} \int_{Q}\left|f(x)-f_{w, Q}\right| w(x) d x<\infty
$$

In the above definition, we have $w(Q)=\int_{Q} w(x) d x$ is the w-measure of $Q$ and the $f_{w, Q}$ is the weighted average given by $f_{w, Q}=1 / w(Q) \int_{Q} f(x) w(x) d x$. The relation between the weights and $\mathrm{BMO}$ is that a function $f$ is in BMO if and only if $f$ is bounded mean oscillation with respect to $w$ for all $w \in A_{\infty}$. Symbolically, we write $B M O=B M O_{w}$ and the norms $\|f\|_{B M O_{w}} \approx\|f\|_{B M O}$. This relationship was proved by Muckenhoupt and Wheeden. Please refer [4] for the detailed proof of these relations.

\section{BMO and Sharp Function Operator}

In this section, we discuss the relation of BMO and sharp function operator. We first begin with the definition of sharp function operator.

\section{Definition:}

Let $\mathrm{f}$ be a locally integrable function defined on $\mathbb{R}^{n}$. A sharp function operator, also known as Fefferman-Stein sharp maximal operator is denoted by $f^{\#}$ or $M^{\#} f$ and is defined as:

$$
f^{\#}(x)=M^{\#} f(x)=\sup _{x \in Q, Q \subset \mathbb{R}^{n}} \frac{1}{|Q|} \int_{Q}\left|f(y)-f_{Q}\right| d y
$$

where $\in \mathbb{R}^{n}$. In the definition, $\mathrm{Q}$ is a cube in $\mathbb{R}^{n}$ with center at $\mathrm{x}$ and sides parallel to the coordinate axes and $f_{Q}$ denotes the average of $f$ over $Q$ given by $f_{Q}=1 /|Q| \int_{Q} f(x) d x$. The difference of the sharp maximal operator compared with the Hardy-Littlewood Maximal function is that instead of the integral averages we maximize the mean oscillation over cubes.

From the definition of sharp function operator, one can show that a function $f$ is a function of bounded mean oscillation, $f \in B M O$ if and only if $f^{\#} \in L^{\infty}\left(\mathbb{R}^{n}\right)$. In other words, we have $\|f\|_{B M O}=\left\|f^{\#}\right\|_{L^{\infty}}$. Moreover, we note that $\left\|f^{\#}\right\|_{L^{\infty}}=0$ if $f \equiv$ constant. Due to this relation,we see that an element in the space of BMO is an equivalence class and two function $f$ and $g$ are equal in BMO if and only if $f-g=$ constant. Under this equality, we see that the space $B M O\left(\mathbb{R}^{n}\right)$ is a normed space. Moreover, we can replace the average $f_{Q}$ by any other constant, say $C_{Q}$ which depends on $Q$. With this we have, a function $f$ is in $B M O\left(\mathbb{R}^{n}\right)$ if and only if for any cube $Q \subset \mathbb{R}^{n}$, there exists a constant $C_{Q}$ depending on $Q$ such that :

$$
\sup _{x \in Q, Q \subset \mathbb{R}^{n}} \frac{1}{|Q|} \int_{Q}\left|f(x)-C_{Q}(x)\right| d x<\infty
$$

Also we have " $f \in B M O\left(\mathbb{R}^{n}\right)$ if and only if $\int_{Q}\left|f(x)-f_{Q}(x)\right| d x<\infty$ " implies that " $f \in B M O\left(\mathbb{R}^{n}\right)$ 
if and only if $\int_{Q}\left|f(x)-f_{Q}(x)\right|^{p} d x<\infty$ for $1 \leq p<\infty$ ". $\quad$ i.e., We have ,

$$
\begin{aligned}
\frac{1}{|Q|} \int_{Q}\left|f(x)-f_{Q}(x)\right| d x & \leq \frac{1}{|Q|} \int_{Q}|f(x)| d x+f_{Q} \\
& \leq 2 \frac{1}{|Q|} \int_{Q}|f(x)| d x
\end{aligned}
$$

for every cube $Q \subset \mathbb{R}^{n}$. Then by the definition of the Hardy-Littlewood maximal function $f^{\#}(x) \leq 2 M f(x)$ for every $x \in \mathbb{R}^{n}$. Moreover, by the maximal function theorem, for every $p$ with $1<p \leq \infty$, there exists constant $C$, depending only on $n$ and $p$ such that:

$$
\left\|f^{\#}\right\|_{p}=2\|M f\|_{p} \leq C\|f\|_{p} .
$$

This shows that the sharp maximal operator is a bounded operator on $L^{p}\left(\mathbb{R}^{n}\right)$ whenever $1<p \leq \infty$. With the help of constant functions, we see that there is no point wise inequality to the reverse direction $M f(x) \leq c f^{\#}(x)$. However, using good lambda inequalities, we can compare the $L^{p}$ norms of the sharp maximal function and the Hardy Littlewood maximal function under certain assumptions. Even though sharp maximal operator and Hardy-Littlewood maximal function are not comparable point wise, they are comparable on $L^{p}$ level. This is given by the following theorem due C. Fefferman and E.M. Stein[5].

Theorem: Let $1<p<\infty$. For every $f \in L^{p}\left(\mathbb{R}^{n}\right)$, there exists a constant $C_{p}$ independent of $f$, depending only on $n$ and $p$, such that:

$$
C_{p}^{-1}\|f\|_{L^{p}} \leq\left\|f^{\#}\right\|_{L^{p}\left(\mathbb{R}^{n}\right)} \leq C_{p}\|f\|_{L^{p}}
$$

and thus $f \in L^{p}\left(\mathbb{R}^{n}\right)$ if and only if $f^{\#} \in L^{p}\left(\mathbb{R}^{n}\right)$.

Using the above theorem, we can show that if $f \in B M O \cap L^{1}$, then we have $f \in L^{p}$, for $1<p<\infty$.

There exist many operators $T$ are bounded from $L^{p}\left(\mathbb{R}^{n}\right)$ to itself for $1<p<\infty$. But this fails for the case $p=$ $1, \infty$. For counter examples please refer Stein-Weiss [6]. The substitute results for the case 1 and $\infty$ are $T: L^{1} \rightarrow$ $L^{1, \infty}$ and $T: L^{\infty, \infty} \rightarrow L^{\infty}$. The substitute result for the action of $T$ on the space $L^{\infty}$ is given by $T: L^{\infty} \rightarrow B M O$, since BMO plays the similar role as that of $L^{\infty}$. Finally, we state a theorem which interpolates $L^{p}$ and BMO and is the extension of the Marcinkiewicz interpolation theorem for the endpoint $(\infty, \infty)$. For the proof, please refer [5].

Theorem: Let $1<p<\infty$ and $T$ be a linear operator, continuous from $L^{p}$ into itself and from $L^{\infty}$ into BMO

$$
T: L^{p}\left(\mathbb{R}^{n}\right) \rightarrow L^{p}\left(\mathbb{R}^{n}\right) \text { and } T: L^{\infty}\left(\mathbb{R}^{n}\right) \rightarrow B M O\left(\mathbb{R}^{n}\right)
$$

continuously. Then $T: L^{q}\left(\mathbb{R}^{n}\right) \rightarrow L^{q}\left(\mathbb{R}^{n}\right)$ continuously for all $p<q<\infty$.

For this and more about the BMO and sharp maximal function, please refer [7].

\section{BMO and Carleson Measure}

To connect the space of BMO with Carleson measure, we first recall the definition of cone and non-tangential maximal functions.

Definition: Let $x \in \mathbb{R}^{n}$. Then a cone over $x$ is given by $\Gamma(x)=(y, t) \in \mathbb{R}_{+}^{n+1}:|x-y|<t$. Let $F: \mathbb{R}_{+}^{n+1} \rightarrow C$. Then the non-tangential maximal function associated to $f$ is defined as:

$$
M^{*} F(x)=\sup _{(y, t) \in \Gamma(x)}|F(y, t)| \in[0, \infty]
$$

For a cube $\subset \mathbb{R}^{n}$, the set given by $T(Q)=Q \times(0, l(Q)]$ is called the tent over the cube $Q$. While solving the famous corona problem, Swedish mathematician L. Carleson [8] obtained the characterization of all non-negative measures $\mu$ defined on $\mathbb{R}_{+}^{2}$ satisfying:

$$
\int_{Q}\left|P_{t} * f(x)\right|^{2} d \mu(x, t) \leq C\|f\|_{L^{2}(\mathbb{R})}^{2}
$$

for all $f \in L^{2}(\mathbb{R})$. Here $P_{t} * f$ is the Poisson integral of $f$ and $C$ is the universal constant.

Now we are state the definition of Carleson measure. Let us take a particular case of characteristic function given by $f=\chi_{2 Q}$. It is easy to see that $\left(P_{t} * f\right)(x) \geq C_{1}$ for some positive constant $C_{1}$. Using this in the above integral inequality, for all $(x, t) \in T(Q)$ we have:

$$
C_{1}^{\prime} \mu(T(Q)) \leq \int_{Q}\left|P_{t} * f(x)\right|^{2} d \mu(x, t) \leq C^{\prime}|Q|
$$

This gives $\mu(T(Q)) \leq C|Q|$. This motivates the following defintion of Carleson measure which was introduced by Carleson in 1960. Please refer [8] for the details. The Carleson measure is closely related with the space of BMO.

Definition: A Carleson measure is a positive measure $\mu$ on $\mathbb{R}_{+}^{n+1}$ such that there exists a constant $C<\infty$ for which $\mu(T(Q)) \leq C|Q|$ for all cubes $Q$ in $\mathbb{R}^{n}$. Here $C$ 
is a universal constant and the smallest of such constant satisfying the inequality $\mu(T(Q)) \leq C|Q|$ is called the norm of the Carleson measure and it is given by

$$
\|\mu\|=\sup _{Q} \frac{\mu(T(Q))}{|Q|}
$$

In the above definition, we can use ball $B(x, r)$ instead of cube $Q$ and we can easily show that the these two definitions are equivalent. We now state the relation between the space of BMO and Carleson measure. This relation was established by $\mathrm{C}$. Fefferman. We state the theorem:

Theorem: The function $g \in B M O\left(\mathbb{R}^{n}\right)$ if and only if the associated measure defined on $\mathbb{R}_{+}^{n+1}$ by

$$
d \mu_{g}(x, t)=y|\nabla u|^{2}(x, t) d x d t
$$

where $u(x, t)=P_{t} * g(x)$ is the Poisson integral of $g$, is a Carleson measure.

There is a decomposition theorem given by Carleson in 1976. Please see [5] for details. We revisit the theorem. Let $\varphi \in C^{1}\left(\mathbb{R}^{n}\right)$ be a radial function which satisfies:

$$
\frac{|\varphi(x)|+|\nabla \varphi(x)|}{(1+|x|)^{n+1}} \leq C \text { and } \int_{\mathbb{R}^{n}} \varphi(x) d x=1
$$

If $h(x)$ is a compactly supported BMO function, then there exists a sequence of functions, say

$\left\{h_{i}\right\}$ such that $\sum_{i=1}^{\infty}\left\|h_{i}\right\|_{L^{\infty}} \leq C\|h\|_{B M O}$ with $C$ is a constant depending only on $n$. In addition to this, there exists a sequence $\left\{\beta_{i}\right\}$ depending only on $x$ such that:

$$
h(x)=h_{1}(x)+\sum_{i=2}^{\infty} \int_{\mathbb{R}^{n}} \varphi \beta_{i}(x-y) h_{i}(y) d y+C_{1}
$$

where $C_{1}$ is a constant. Conversely, if a function $\mathrm{h}$ has the above decomposition, then the function $h \in B M O\left(\mathbb{R}^{n}\right)$ and the norm of $h$ satisfies:

$$
\|h\|_{B} M O \leq C \sum_{i=1}^{\infty}\left\|h_{i}\right\|_{L^{\infty}}
$$

Finally the readers are suggested to refer [6] for more about the relation between BMO and Carleson measure and various characterization of the space of functions of bounded mean oscillations.

\section{References}

[1] F. John and L. Nirenberg, "On functions of bounded mean oscillation," Comm. Pure Appl. Math, vol.14, pp. 415-426, 1961.

[2] C. Feffereman, "Characterization of bounded mean oscillations," Bulletin of American Mathematical Society, vol.77, 1971.

[3] L. Grafakos, "Modern Fourier Analysis, Graduate texts in mathematics", 3rd ed. New York, 2014.

[4] J. Gracia-Cuerva and J.-L. Rubio de Francia, "Weighted norm inequalities and related topics", North-Holland Mathematics Studies, 116, Notes de Matematica, vol. 104, 1985.

[5] C. Fefferman and E.M. Stein, "Hp spaces of several variables", Acta. Math, vol. 129, pp. 137-588, 1972.

[6] E. M. Stein and G. Weiss, 'Fourier Analysis on Euclidean Spaces. Princeton University Press, New Jersey, 1971.

[7] D. Chang and C. Sadosky,"Functions of Bounded Mean Oscillation," Taiwanese Journal of Mathematics, vol. 10, no. 3, pp 573-601, 2006.

[8] L. Carleson, "Interpolation of bounded functions and the corona problem", Ann. Mathematics, vol. 76, pp. 547-559, 1962. 
